\title{
BEAM-BEAM SIMULATION USING THE UNIFIED ACCELERATOR LIBRARIES
}

\author{
T. Koyama, N. Malitsky and R. Talman \\ Laboratory of Nuclear Studies, Cornell University, Ithaca, NY 14853 USA
}

\begin{abstract}
Colliding beam experiments at CESR with beams made round by running on the coupling resonance have been performed[1], and runs are planned with the beams made round by Möbius modification[2]. To simulate performance in these configurations stochastic and beam-beam modules have been added to the Unified Accelerator Libraries[3] for use along with pre-existing lattice representation, and map and element-by-element, TEAPOT++ modules. For a realistic CESR lattice containing sextupoles beam-beam performance is simulated.
\end{abstract}

\section{INTRODUCTION}

Over the years, many accelerator simulation programs have been developed. These programs, libraries and data formats tend to be inoperable with one another, and major modifications are required for them to work together. To cope with this interoperability issue, the Unified Accelerator Library (UAL) has been designed and developed[3].

The UAL not only gives guidelines to programmers who develop codes in the future but also enables existing "legacy" codes to work seamlessly with other UAL compliant codes. In other words, with one-time-only modifications, old codes can be integrated with other UAL compliants. Due to the UAL, the lifetime of codes is expected to be extended considerably, permitting more emphasis to be placed on design and algorithms.

One of the objectives of this paper is to demonstrate the effectiveness and versatility of the UAL to enable realistic simulation. The other objective is to predict the luminosity of CESR. To achieve the latter, real CESR lattice data is extracted from the CESR database and converted into Standard Machine Format (SMF)[4], and a beam-beam interaction UAL module and a stochastic-damping[5] UAL module have been developed to simulate the essential beam physics.

\section{SIMULATION}

To simulate colliding beams, one beam is described by an analytical distribution function and the other is described by a large number of macroparticles. As the macroparticles are tracked, self-consistency is achieved by allowing the parameters of the analytic distribution to evolve. A flow diagram of the simulation is shown in Fig. 1.

First, we have to supply input parameters relevent to the CESR operations. These values are either obtained empirically from direct measurements, or from computations of other computer programs, or as control parameters of the

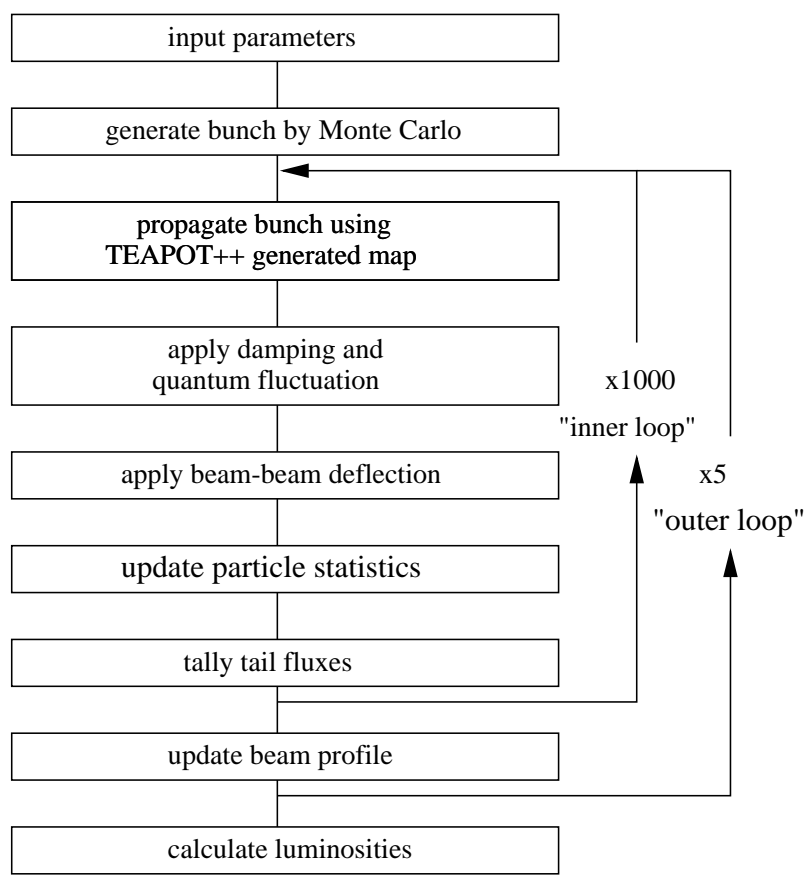

Figure 1: Program flow diagram.

simulation. The empirical ones include values such as energy deviation, damping coefficient, average energy, and bunch length. The computed values include Twiss parameters and the CESR lattice description. The only control parameter we have used so far in this simulation is beam current.

Next, a single bunch with, say 1000 , sample particles is Monte Carlo generated in accordance with the input parameters. Parameters of this artifical bunch are preferably in the neighborhood of the "self-consistent" state for faster convergence.

After the bootstrapping processes, the program enters nested loops. The "outer loop", associated with beam evolution, consists of $1000^{1}$ (typically) cycles of "inner loops". In the inner loop, we transport the beam around CESR by the TEAPOT++ generated map, apply damping and quantum fluctuation, apply beam-beam interaction, update particle statistics, and tally tail fluxes of the beam. This whole outer loop cycle is iterated about 5 times until a selfconsistent state is reached.

The first process in the inner loop is particle propagation around the CESR lattice. Since the TEAPOT++ elementby-element tracking method is considerably slower than the

\footnotetext{
${ }^{1}$ The number of cycles should be appreciably larger than the inverse of the damping coefficient to achieve equilibrium
} 
map method, we employed the map method in the simulation. However, this map method has to be checked. To do so, we transported a particle with initial amplitude $1 \mathrm{~mm}$ at the interaction point using element-by-element tracking, 6 th order map, 8th order map, and 10th order map. The displacements after 5000 turns are tabulated in Table 1. The results show that even 6th order can provide accurate propagation. Since essentially all particles lie within $1 \mathrm{~mm}$ from the center at the interaction point as seen in Fig. 3, it is valid to use the map method to transport the particles in the storage ring for faster processing. In particular, we have chosen an 8 th order map ${ }^{2}$.

\begin{tabular}{lllll} 
method & $x$ & $x^{\prime}$ & $y$ & $y^{\prime}$ \\
\hline track & 9.4828478 & 3.8684952 & 8.2396275 & 1.4646015 \\
map6 & 9.4904625 & 3.8555656 & 8.2165072 & 1.4473738 \\
map 8 & 9.4831417 & 3.8681267 & 8.2392599 & 1.4643757 \\
map10 & 9.4828516 & 3.8684907 & 8.2396238 & 1.4645996
\end{tabular}

Table 1: Displacements of various transporting methods after 5000 turns with initial amplitude $1 \mathrm{~mm} . x$ and $y$ are in units of $10^{-4} \mathrm{~m}$, and $x^{\prime}$ and $y^{\prime}$ are in $10^{-3}$.

Comparison among maps of different orders are tabulated in Table 2. The numbers in the second and the third columns are calculated by fomulas:

$$
\sum_{k=1}^{\text {order }} \frac{(k+\operatorname{dim}-1) !}{k !(\operatorname{dim}-1) !}
$$

and

$$
\sum_{k=1}^{\text {order }} \frac{(k+\operatorname{dim}-1) !}{k !(\operatorname{dim}-1) !}(k-1)
$$

respectively ${ }^{3}$. These tables provide information useful for selection of an optimal method and to estimate processing times.

\begin{tabular}{llll} 
order & $\begin{array}{l}\text { generation } \\
\text { time }\end{array}$ & terms & multiplications \\
\hline 6 & $3 \mathrm{~min}$ & 923 & 3829 \\
8 & $25 \mathrm{~min}$ & 3002 & 17590 \\
10 & $150 \mathrm{~min}$ & 8007 & 60633
\end{tabular}

Table 2: Comparisons among map methods. The times are measured on a system with SPECInt92 $\approx 200$.

Next, damping and quantum fluctuation is applied to a once-circulated bunch. The damping decrement we used so far is 10 times larger than the empirical value for faster convergence. And the amplitude of quantum fluctuation is:

$$
\sqrt{12 \delta_{d}} \sigma_{0}
$$

where $\delta_{d}$ is damping constant. This amplitude is multiplied by a uniformly distributed random number between -1 and

\footnotetext{
${ }^{2}$ Because of synchrotron radiation damping, preservation of symplecticity is not a critical issue.

${ }^{3}$ In our case, $\operatorname{dim}=6$
}

1. The quantum fluctuation is applied in both horizontal and vertical directions with independent random numbers.

After damping and quantum fluctuation, we apply beambeam deflection to a bunch. The deflection due to the beambeam interaction is calculated by the formula:

$$
\Delta p_{x}, \Delta p_{y}=-2 r_{e} \frac{m_{e}}{E} N C_{r} \frac{x, y}{r^{2}}
$$

where $C_{r}$ is a (cumulative) circularly symmetric distribution, $r_{e}$ is the classical radius of electron, and $\mathrm{N}$ is the total number of particles in a bunch. During the first 1000 turns, the beam-beam deflection is calculated with a Rayleigh distribution (which is just a circularly symmetric Gaussian distribution in polar coordinates) with a standard deviation consistent with the input Twiss parameters. However, starting from the second outer loop cycle, a "near-Rayleigh distribution" " obtained at the end of previous outer loop cycle is used to calculated beam-beam deflections until the end of the cycle. This corrected beam profile gradually drives the beam into a self-consistent state.

Then, we update the particle distribution statistics and this accumulated data is used to fit the beam profile with an improved "near-Rayleigh probability density function" at the end of every outer loop cycle ${ }^{5}$ :

At the end of every inner loop, we take statistics of particles crossing preset boundaries located at $8 \sigma, 10 \sigma, 12 \sigma$, and $14 \sigma$ in phase space, where $\sigma$ is the initial CourantSnyder invariant. To prevent multiple counting of particles wandering near boundaries, we put "Schmidt trigger" like buffer layers of $\frac{1}{2} \sigma$ surrounding those boundaries. Those crossing the $14 \sigma$ boundary are declared lost and are restarted in accordance with the initial parameters. These data are used to estimate lifetimes.

After 1000 inner loop repetitions, we use the collected particle distribution data to fit the beam profile by nearRayleigh function. The first and the second moments of the distribution are used to calculate power $p$ and $\sigma_{n r}$ as in the equation (5) by a formula:

$$
\frac{\langle r\rangle^{2}}{\left\langle r^{2}\right\rangle}=\frac{\Gamma^{2}(3 / p)}{\Gamma(2 / p) \Gamma(4 / p)}
$$

and

$$
\sigma_{n r}=2^{-1 / p} \frac{\Gamma(2 / p)}{\Gamma(3 / p)}\langle r\rangle
$$

Finally, after the nested loop processes, luminosity of a self-consisted state is calculated from the near-Rayleigh function of the self-consistent state.

${ }^{4}$ The corresponding probability density function is:

$$
P_{R_{p}}\left(r, \sigma_{n r}\right)=\frac{p}{2^{2 / p} \sigma_{n r}^{2} \Gamma(2 / p)} r e^{-\frac{1}{2}\left(\frac{r}{\sigma_{n r}}\right)^{p}} .
$$

This near-Rayleigh function is a generalization of the well-known Rayleigh probability density function.

${ }^{5}$ Data in the database is cleared at the end of each outer loop cycle 


\section{RESULTS}

This whole simulation is repeated for several beam strengths from $1 \mathrm{~mA}$ through $28 \mathrm{~mA}$ as explained in the previous section. Obtained luminosities are plotted against beam current in Fig. 2. As beam currents increase, deviations from the quadratic single beam curve become large due to beam-beam interaction. The quadratic curve is based on the $1 \mathrm{~mA}$ point, where the beam-beam effect is insignificant.

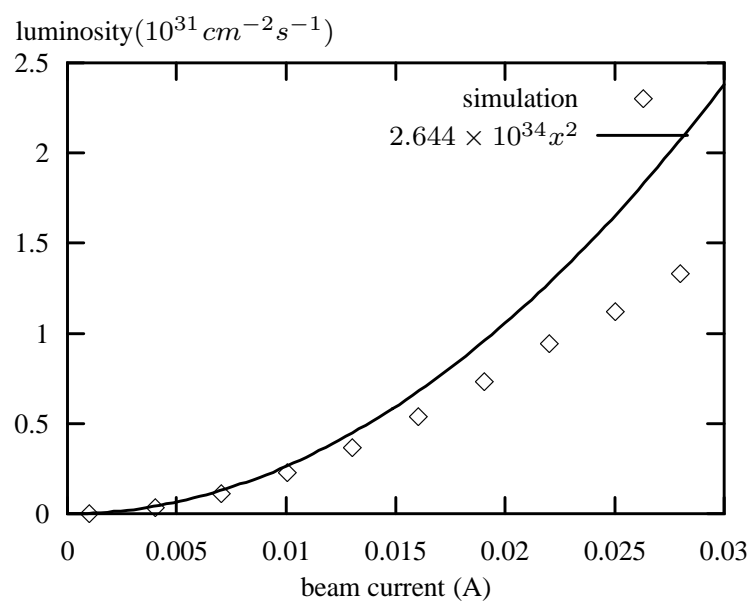

Figure 2: Graph of luminosity versus beam current.

It is also interesting to see how the beam profile evolves as the beam current increases. To see the beam evolution, we have plotted the fitted near-Rayleigh density functions of those self-consistent states in Fig. 3. As can be seen in the figure, tails of the beam profile grow, the peaks move outward, and entire beam profiles are correspondingly lowered somewhat as beam intensity increases.

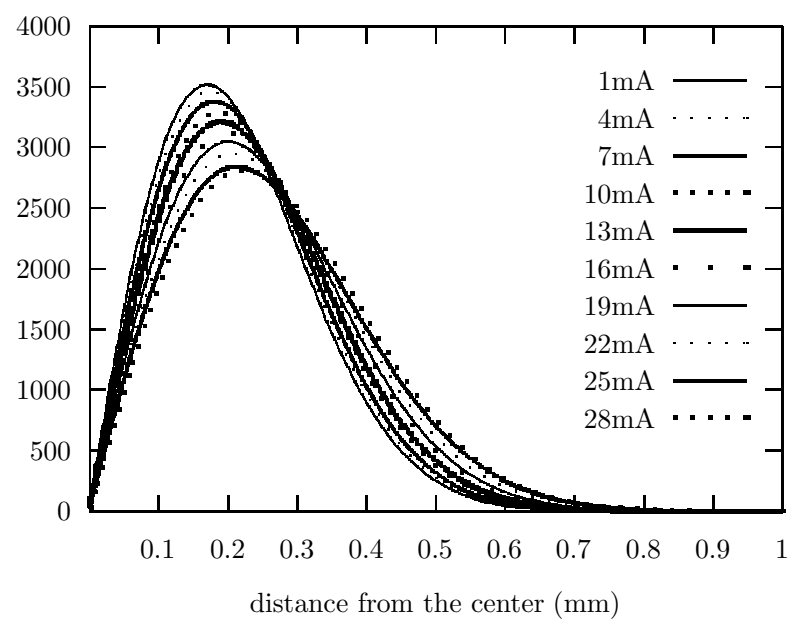

Figure 3: Self-consistent states of various beam currents at $\beta \approx 0.2 \mathrm{~m}$.

Lifetimes of high beam currents are estimated based only on the final outer loop cycle. This is because most particle loss takes place during evolution to the self-consistent state, but we are only interested in the stability of the selfconsistent state. To estimate lifetimes, we fit the plot of emittance versus logarithm of number of boundary crossings with a straight line. This is then extraporated to various aperture sizes from $14 \sigma$ through $20 \sigma$. The result is tabulated in Table 3. These estimates cannot be expected to have absolute values, but their relative significance as other parameters vary is expected to help finding optimal operating conditions.

\begin{tabular}{lllll} 
current & $14 \sigma$ & $16 \sigma$ & $18 \sigma$ & $20 \sigma$ \\
\hline $22 \mathrm{~mA}$ & $10 \mathrm{~s}$ & $10^{2} \mathrm{~s}$ & $10^{3} \mathrm{~s}$ & $10^{4} \mathrm{~s}$ \\
$25 \mathrm{~mA}$ & $1 \mathrm{~s}$ & $10 \mathrm{~s}$ & $10^{2} \mathrm{~s}$ & $10^{2} \mathrm{~s}$ \\
$28 \mathrm{~mA}$ & $1 \mathrm{~s}$ & $1 \mathrm{~s}$ & $10 \mathrm{~s}$ & $10 \mathrm{~s}$
\end{tabular}

Table 3: Estimated beam lifetime for high beam current.

\section{PLANS AND PROSPECTS}

We intend to develop a method to apply the quantum fluctuation process collectively rather than turn-by-turn as in this simulation. Such a method will enable us to construct a multiple turn map with beam-beam interaction and stochastic-damping process incorporated. Furthermore, a semi-continuous model of the beam may replace the discrete particle model employed in this simulation.

\section{REFERENCES}

[1] E. Young et al. Collisions of resonantly coupled round beams at the cesr. In Proceedings to this conference, (1997).

[2] R. Talman. Phys. Rev. Lett., 74, 1590 (1995).

[3] N. Malitsky and R. Talman. Status of unified acclerator libraries. In Proceedings to this conference, (1997).

[4] N. Malitsky et al. A proposed flat yet hierarchical accelerator lattice object model. Particle Accelerators, 55, 313 (1996).

[5] E. Young and R. Talman. Design, analysis, and compensation of coupled accelerator lattices. CESR internal report, (1997). 